\title{
Preface to the special issue: nanomaterials, scope, synthesis, and applications
}

\author{
Sofian Kanan
}

Published online: 7 August 2011

(C) Springer Science+Business Media B.V. 2011

Nanotechnology is of great interest to scientists and engineers because nano-sized particles exhibit different properties from both individual atoms and bulk properties in a material. Over the last three decades, scientists have investigated the whole spectrum of nanomaterials, which covers synthesis protocols, materials characterization, as well as surface and optical properties. Materials including metallic, metal oxides, semiconducting composites, polymeric nanostructured surfaces, and carbon nanotubes have been investigated. Understanding both the physical and chemical characteristics of the materials leads to the fabrication of various nano-based devices for numerous applications including size- or shapeselective catalysis, semiconducting metal oxide-based sensors for the detection of gaseous pollutants, biological sensors, gas separation and purification, electrochemical devices, nanoenergetics, nanoelectronics, and photonics, as well as magnetic detection.

Because of the variety of topics covered on nanomaterials in terms of pore structure with designed morphology, compositional variations, surface properties, and functionalities, there is a great deal of interest in materials modification to tailor unique and stable nanomaterials for specific applications. Active scientists and engineers with primary research interests focused on nanomaterials toward various applications are invited to contribute to this special issue with the ultimate goal of promoting the growing interest in this developed and applied field.

\footnotetext{
S. Kanan $(\bowtie)$

Department of Biology and Chemistry, American University of Sharjah, Sharjah, UAE e-mail: skanan@aus.edu
} 